\title{
Development of Brain Structures, Connections, and Functions
}

\author{
Edith V. Sullivan
}

Received: 11 October 2010 /Accepted: 11 October 2010/Published online: 19 October 2010

(C) Springer Science+Business Media, LLC 2010

Concepts regarding structural and functional development of the brain have changed dramatically over the last generation. The birth and death of neurons as a normal developmental process in utero and post partum allow for significant influence of environmental interaction in modeling brain development. In this issue of Neuropsychology Review, six papers, written by researchers with extensive expertise in different areas of brain development, provide evidence of the fragility, plasticity, and resilience of the developing brain. Estimation and tracking of neurodevelopmental changes are aided by a variety of neuroimaging modalities that enable safe, longitudinal examination of changing brain structure (e.g., Fig. 1) and function. The astounding importance that early childhood experiences has on neuropsychological and psychiatric health cannot be underestimated and underscores the value of early nurturing, emotional interchange, and energetic engagement in educational experiences.

Joan Stiles and Terry Jernigan present a comprehensive and lucid review of the basics of brain development from biological, neuroimaging, and neuropsychological perspectives. The authors remind us of the terms "experience expectant" development and "experience dependent" learning as central constructs underlying individual variation in development and the potential for improving experience during critical periods of development. This theme pervades the issue, highlighting the importance of environmental experience during critical periods of development.

\section{E. V. Sullivan $(\bowtie)$}

Department of Psychiatry \& Behavioral Sciences,

Stanford University School of Medicine,

401 Quarry Road,

Stanford, CA 94305-5723, USA

e-mail: edie@stanford.edu
Jay Giedd, Michael Stockman, Catherine Weddle, Maria Liverpool, Aaron Alexander-Bloch, Gregory Wallace, Nancy Lee, Francois Lalonde, and Rhoshel Lenroot relate findings from longitudinal studies of children and adolescents supportive of extensive post-natal morphological changes in the developing brain. A pattern that has emerged from studies using structural imaging, sleep electrophysiology, and positron emission tomography has been confirmed with longitudinal investigation providing evidence for growth of cortical gray matter volume until about age 5 to 10 years, followed by an inexorable decline through to old age. White matter volume increases throughout adolescence, and possibly much longer, and provides the essential infrastructure for communication across long distances in the brain. Twin studies provide evidence for genetic factors contributing to a significant portion of brain growth variance.

Alecia Vogel, Johathan Power, Steven Petersen, and Bradley Schlaggar describe methods and findings based on noninvasive, in vivo neuroimaging approaches to identify and track functional connectivity in the developing brain. One general principle these authors identified is a developmental difference between children and adults in their basic organization of brain networks. Whereas networks of adults are distributed throughout the brain, those of children are organized by anatomical proximity.

Margaret Sheridan, Stacy Drury, Kate McLaughlin, and Alisa Almas remind us of the importance that early childhood experiences and nurturing contribute to cognitive and emotional development in the context of neurobiological development. They recount that the experience of children previously institutionalized in foster or adoptive care can determine adult outcome, especially in cases where environmental, nutritional, or emotional input was tenuous during development. The end result we observe with adult 


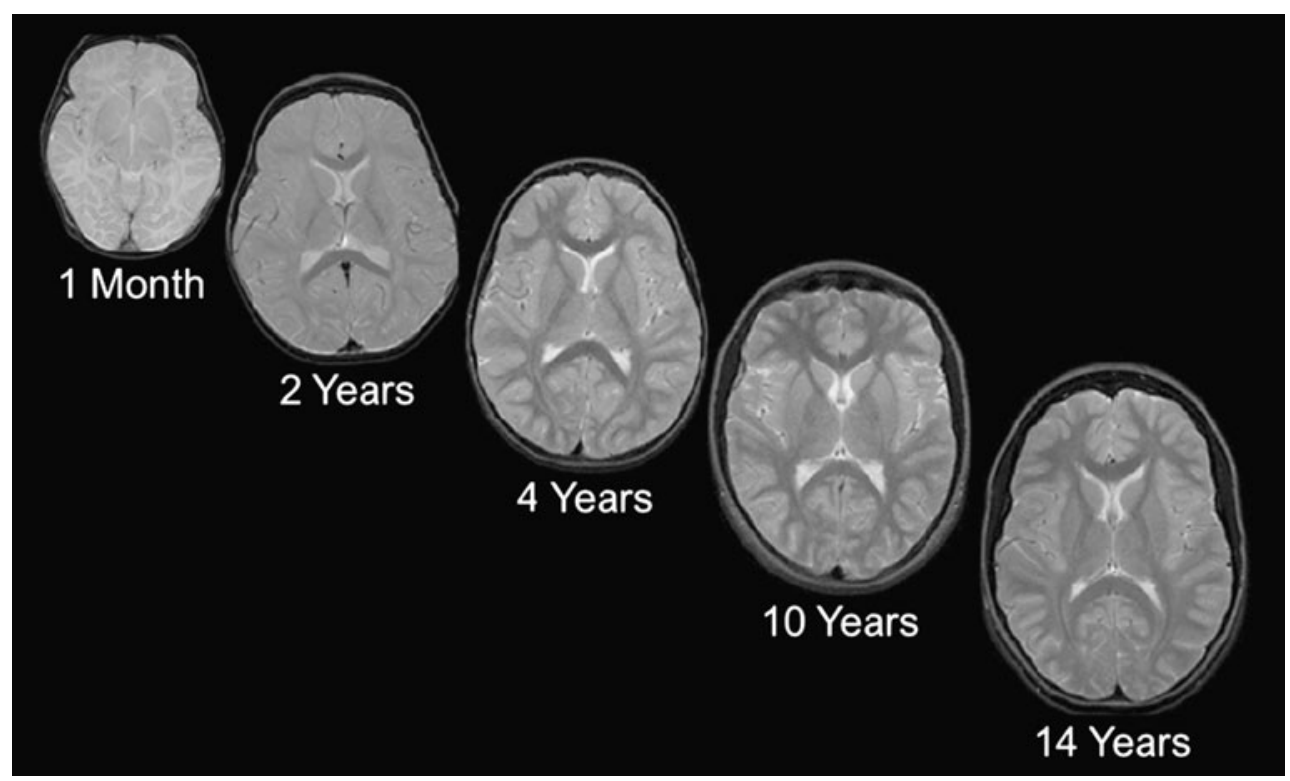

Fig. 1 Magnetic resonance images of five young, healthy individuals, age 1 month to 14 years. Notable changes in morphology include enlargement of intracranial and ventricular volume and change in conspicuity of tissue types on proton density images. For example, before age 1 year, white matter is generally unmyelinated and appears brighter than gray matter because of the higher water content in unmyelinated white matter compared with myelinated white matter or

neuropsychological testing can be determined in large part by early childhood experience - or lack thereof.

Florence Roussotte, Linsay Soderberg, and Elzabeth Sowell review neuroimaging findings on prenatal exposure to alcohol, cocaine, and methamphetamine. Each agent affects different constellations of brain systems, and the extent of effect depends on timing of prenatal exposure. Knowledge about these potentially hidden effects could explicate some behavioral, cognitive, and motor deficits that manifest during development and young adulthood.

Sunita Bava and Susan Tapert show that the brain continues structural development throughout adolescence, a gray matter. After 1 year of age, myelination proceeds, causing myelinated white matter, with its high lipid content, to appear darker than gray matter, which has higher water concentration than myelinated white matter. Images were produced courtesy of Adolf Pfefferbaum, M.D., Director of the Neuroscience Program, SRI International and Professor Emeritus, Stanford University School of Medicine

time when youth test the waters of life and characteristically engage in risky behavior. Along with this remarkable plasticity is vulnerability to environmental teratogens, including legal and illicit substances of abuse - alcohol, marijuana, cocaine, and other psychoactive agents. Whether neurofunctional changes that occur with alcohol and drug abuse and dependence in youth are reversible remains unknown.

Acknowledgment This work was supported by grants from the NIAAA (AA010723, AA017168, AA017923) and NIA (AG017919).

Disclosures The author is the editor-in-chief of the journal. 